\title{
Epidemiology, Biochemistry and Evolution of Trypanosoma cruzi Lineages Based on Ribosomal RNA Sequences
}

\author{
Bianca Zingales $/{ }^{+}$, Beatriz S Stolf, Ricardo P Souto $/{ }^{++}$, Octavio Fernandes*, \\ Marcelo RS Briones**
}

\begin{abstract}
Departamento de Bioquímica, Instituto de Química, Universidade de São Paulo, Caixa Postal 26077, 05599-970 São Paulo, SP, Brasil *Departamento de Medicina Tropical, Instituto Oswaldo Cruz, Rio de Janeiro, RJ, Brasil **Departamento de Microbiologia, Imunologia e Parasitologia, Escola Paulista de Medicina, Universidade Federal de São Paulo, São Paulo, SP, Brasil
\end{abstract}

Key words: Trypanosoma cruzi - major lineages - ribosomal RNA - molecular epidemiology - phylogeny

A puzzle in Chagas disease research has been to correlate molecular markers with epidemiology since isolates of Trypanosoma cruzi exhibit a broad host range, induce distinct clinical presentations in patients and show great diversity in biological and biochemical characteristics. Early studies on population genetics revealed substantial isozymic variability among isolates of $T$. cruzi defining three major groups or zymodemes named Z1, Z2 and Z3 (Miles et al. 1978, 1980). Further analysis of 15 gene loci disclosed a greater heterogeneity determining the distribution of $121 \mathrm{~T}$. cruzi isolates into 43 zymodemes that could not be grouped by the authors in few natural clusters (Tibayrenc \& Ayala 1988). The main conclusion of these studies was that the population structure of this parasite is clonal rather than sexual, and, as a consequence, the present genetic and biological variability of $T$. cruzi is resultant from the independent evolution of clonal lines (Tibayrenc \& Ayala 1988). Acceptance of clonal propagation of $T$. cruzi implies that the classical definition of species is not fully adequate to this parasite. It has been proposed that each distinct genotype should be studied individually and special attention given to the most represented ones (Tibayrenc 1995). On the other hand, other groups still believe that it is worth searching for genetically and ecologically coherent group-

\footnotetext{
This work was supported by Fundação de Amparo à Pesquisa do Estado de São Paulo (Fapesp) and Conselho Nacional de Pesquisa (CNPq).

${ }^{+}$Corresponding author. Fax: + 55-11- 815.5579. E-mail: bszodnas@quim.iq.usp.br

${ }^{++}$Present address: Department of Biochemistry, Boston University School of Medicine, 80 East Concord St., Boston, MA 02118, USA

Received 9 June 1999

Accepted 9 August 1999
}

ing of isolates (Macedo \& Pena 1998). Indeed the remarkable genetic diversity of $T$. cruzi (Morel et al. 1980, Henriksson et al. 1990, Macedo et al. 1992) initially identified could be somewhat organized (Henriksson et al. 1993, Tibayrenc 1995). However, analyses of these fast evolving genetic markers could not clearly show defined clusters.

\section{TYPING OF T. CRUZI ISOLATES BASED ON A RRNA SEQUENCE}

In contrast to the diversity suggested by former approaches, analyses of markers with lower evolutionary rate such as multigenic families suggested dichotomy within $T$. cruzi isolates. PCR amplification of a sequence from the D7 divergent domain of the 24Sa rRNA (LSU) gene indicated dimorphism among T. cruzi isolates and allowed the division of 16 parasite stocks into two groups (Souto \& Zingales 1993). This observation was confirmed by others following riboprinting analysis in 18 isolates from North America (Clark \& Pung 1994). Subsequently, a variable spot in the spacer region of the mini-exon gene was found in strains of T. cruzi which also showed dimorphism after PCR amplification (Souto et al. 1996).

The typing approaches based on rRNA and mini-exon sequences were used to analyse $88 T$. cruzi stocks from humans, insects and sylvatic animals from several Latin America countries. Amplification of these sequences originated 125 or $110 \mathrm{bp}$ products for rDNA and 300 or $350 \mathrm{bp}$ products for the mini-exon. Within individual isolates, one of the three associations was observed: the $125 \mathrm{bp}$ rDNA product with the $300 \mathrm{bp}$ mini-exon product (defined as group 1), the 110bp rDNA product with the $350 \mathrm{bp}$ mini-exon product (defined as group 2), and the presence of both rDNA amplification products with the mini-exon group 1 product (defined as group 1/2) (Fig. 1) (Souto et al. 1996). RAPD analysis showed variability between individual isolates, however, tree analysis clearly indicated the presence of two major 
branches. Interestingly, the group 2 isolates correlated precisely with one branch of the tree, and group 1 and group 1/2 isolates correlated with the other branch (Fig. 1) (Souto et al. 1996). Our studies clearly indicate that $T$. cruzi isolates can be partitioned into two major Lineages, which we named Lineage 1 and 2 (Fig. 1) (Souto et al. 1996). In that study several isolates classified as Z1 and Z2 were analyzed and it was concluded that Z2 stocks belong to Lineage 1, while stocks classified as Z1 fall into Lineage 2. The position of Z3 was further investigated based on mini-exon sequence analysis and it was concluded that it constitutes a discrete sub Lineage within Lineage 2 (Fernandes et al. 1998a). This observation agrees with previous conclusions indicating that $\mathrm{Z3}$ although distinct from, is related to Z1 (Miles et al. 1980).

The existence of these lineages can be noticed by detailed isoenzyme data (Tibayrenc 1995). Concerning the major clones defined by Tibayrenc and Ayala (1988), our typing assays indicate that clones $19 / 20$ belong to Lineage 2, while clones 32 and 39 belong to Lineage 1 . It should be pointed out that clone 39 maintains heterozygosity at several loci and is classified as group 1/2 (Souto et al. 1996). More recently, the bi-polar grouping of T. cruzi has been supported by riboprinting analysis (Stothard et al. 1998), rRNA and mini-exon (spliced-leader) promoter sequences and activities (Floeter-Winter et al. 1997, Nunes et al. 1997a, b), microsatellite markers (Oliveira et al. 1998) and structure of ribosomal spacers (Fernandes et al. 1999a).

Presently, several laboratories are involved in the definition of the relevance of the two lineages and their sub-groups with respect to the epidemiological and biological properties of T. cruzi. These studies as well as the analysis of the evolutionary origin of the two Lineages should indicate whether each Lineage constitutes, according to the modern concept of species, an ecological and a genetic unit (Macedo \& Pena 1998).

\section{THE RIBOSOMAL RNA CISTRON}

Southern blot analysis (Souto et al. 1996), riboprinting (Clark \& Pung 1994, Stothard et al. 1998) and restriction profiles of internal transcribed spacers (ITS) from the rDNA cistron (Fernandes et al. 1999a) clearly indicate that the overall structure of the ribosomal RNA cistron differs between isolates of Lineage 1 and Lineage 2, supporting this division. It has also been shown that group 1/ 2 isolates have both types of rRNA cistrons, with eight to ten-fold greater copy number of group 2rDNA genes relative to group 1-rDNA genes (Souto et al. 1996).

Previous studies in different organisms have shown that rRNA gene promoters exhibit species- specific control (Sollner-Webb \& Towers 1986). A plasmid construct bearing the promoter sequence from the rRNA cistron of the CL strain drove the expression of chloramphenicol acetyl transferase (CAT) when transfected into epimastigotes of some T. cruzi strains, but surprisingly, was inactive in others (Tyler-Cross et al. 1995). We examined the activity of the same construction in isolates belonging to the two major phylogenetic lineages. It was observed that CL strain (Lineage 1) rRNA promoter drove high CAT activity in Lineage 1 isolates, but essentially no activity in Lineage 2 strains. Thus, the CL rRNA promoter exhibited a clear Lineage 1-specificity (Floeter-Winter et al. 1997, Nunes et al. 1997a).

In order to analyse the activity of Lineage 2 rRNA promoter, we cloned the corresponding segment from the Dm28 strain. Alignment of this sequence $(800 \mathrm{bp}$ ) with rRNA promoters from other strains of T. cruzi indicated $98 \%$ and $82 \%$ identity with sequences of Lineage 2 and Lineage 1 strains, respectively (Floeter-Winter et al. 1997, Stolf 1999). These data indicate that the sequence homology in this region also reflects the division into two lineages. We have also analysed the rRNA promoter sequences from group $1 / 2$ isolates, which contains two types of ribosomal RNA cistrons. It was observed that the type 1 promoter sequence of NR cl3 clone (group 1/2) has $96 \%$ identity with that of CL Brener clone (Lineage 1, group 1) and $80 \%$ identity with that of Dm28 strain (Lineage 2, group 2). On the other hand, the type 2 promoter sequence of NR cl3 clone has $82 \%$ identity with that of CL Brener clone and $90 \%$ identity with that of Dm28 strain (Stolf 1999).

The activity of Lineage 1 and Lineage 2 promoters was investigated by transient expression of CAT reporter gene in different $T$. cruzi isolates typed as rDNA group 1, group 2 and group $1 / 2$ (cf in Fig. 1). It was observed that Lineage 1 promoter is expressed in both group 1 and group $1 / 2$ strains, but shows no activity in group 2 isolates. On the other hand, Lineage 2 promoter is expressed in the three groups of isolates (Nunes et al. 1997a, Stolf 1999). In the specific case of group $1 / 2$ it was observed that the expression of CAT driven by L2 promoter was higher than that driven by the L1 promoter. Interestingly, RT-PCR experiments conducted with total RNA extracted from group 1/2 isolates allowed to conclude that only the rRNA cistron of type 2 is transcribed in vivo (Stolf 1999).

Taken together, our data indicate that there are clear differences in the sequence and activity of the rRNA promoter regions in the representatives of the two major lineages. The specificity of the promoter activity is not mutually exclusive, since group 1 and group 1/2 strains express promoters 


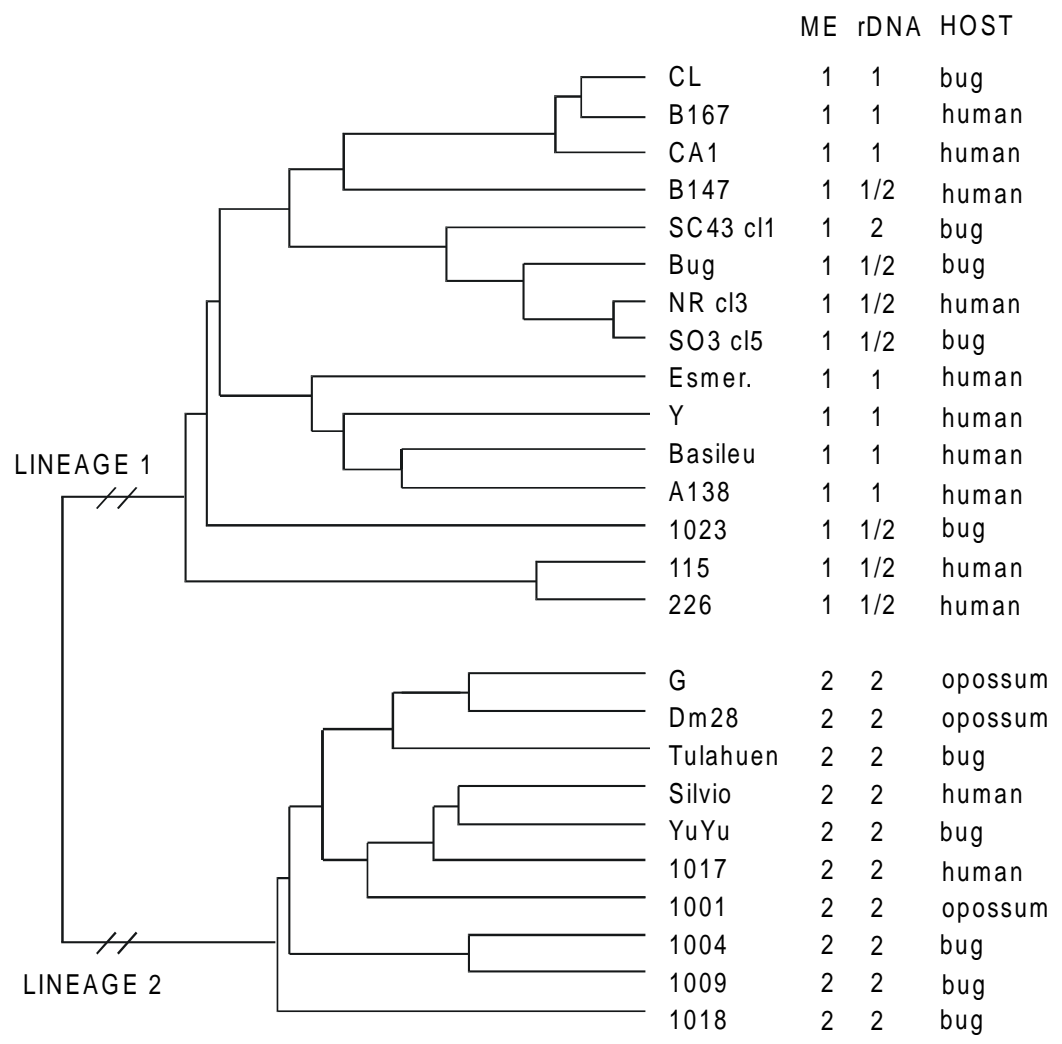

Fig. 1: UPGMA tree based on the proportion of bands not shared among the isolates. For each isolate the corresponding rDNA and mini-exon groups as well as the host from which it was derived are indicated (after Souto et al. 1996).

from both groups, but group 2 strains only express group 2 promoters. The reasons for these observations are unclear but might be related to the characteristics of the components of the transcription machinery in the T. cruzi groups. Nevertheless, since it has previously been shown for many eukaryotes that the rRNA promoter activity is species-selective (Sollner-Web \& Towers 1986) the observation that $T$. cruzi rRNA promoters show group-specificities supports the conclusion that these groups could be considered discrete taxa.

\section{EPIDEMIOLOGICAL DISTRIBUTION}

We have analysed by the 24Sa rRNA and/or mini-exon typing methods more than 200 isolates from triatomine species and mammalian hosts from the domestic and sylvatic cycles originating from 12 Brazilian states. Data provide evidence for a strong association of $T$. cruzi Lineage 1 with the domestic cycle, while Lineage 2 is preferentially encountered in the sylvatic cycle (Fernandes et al. 1998b, 1999b, Zingales et al. 1998, cf also Fig. 1). Data also suggest that Lineage 1 has properties that favour human infections, possibly due to higher parasitemia, since all parasites isolated from seropositive individuals from endemic regions be- long to this Lineage (Fernandes et al. 1998b, Zingales et al. 1998). On the other hand, Lineage 2 was isolated from very few human seropositive cases in Amazonas where T. cruzi is enzootic (Zingales et al. 1998).

These observations concerning the general behaviour of the two lineages in chagasic patients in Brazil agree with data regarding the distribution of two major clones: 20 (Lineage 2) and 39 (Lineage 1) in children and vectors from a Bolivian endemic area (Breniere et al. 1998). It was concluded that clone 39 is prevalent in patients, while in vectors (Triatoma infestans) clones 20 and 39 are found with comparable frequencies. These results suggest a limited selection in the transmission of the two clones and a drastic control of clone 20 parasitemia by the immune system of children patients.

Analysis of 68 T. cruzi stocks from mammals and triatomines of the Atlantic Coast rainforest in the State of Rio de Janeiro suggests a preferential adaptation of Lineage 1 to primates and Lineage 2 to opossums (Fernandes et al. 1999b), as has also been concluded from studies conducted in Georgia State (USA) (Clark \& Pung 1994, Pung et al. 1998). 


\section{BIOLOGICAL EVIDENCE}

Several biological evidence also support the conclusion that the two phylogenetic lineages have different characteristics. Experimental infection of marsupials with strains of Lineages 1 and 2 indicates the elimination of type 1 strain from the host, while the type 2 strain promotes a permanent and very mild infection (Carreira et al. 1996). In vivo and in vitro experiments suggest that the two lineages differ markedly in infectivity to mammals. In fact, it has been shown that two T. cruzi strains $\mathrm{Y}$ and CL (Lineage 1) are highly infective to mice, while strain $\mathrm{G}$ (Lineage 2) does not produce parasitemia (Yoshida 1983). This observation agrees with data derived from the analysis of isolates from human chagasic patients (Fernandes et al. 1998b, Zingales et al. 1998). It has also been reported that Lineage 1 strains are four times more infective to HeLa cells than Lineage 2 strains. These results have been correlated with the expression of glycoprotein gp90 which seems to inhibit calcium mobilization required for cell invasion and is present in the eight analyzed strains of Lineage 2 but not in Lineage 1 isolates (Ruiz et al. 1998). Regarding the parasite-triatomine vectors interaction, it is known that growth and metacyclogenesis of different $T$. cruzi isolates in the insect depend on the strain of the parasite (Garcia \& Azambuja 1991, Lana et al. 1998). For example, it has been shown that infection of Dipetalogaster maximus with two different clones of $T$. cruzi showed that only one clone underwent metacyclogenesis. Similar results were seen when different strains and clones of $T$. cruzi infected Rhodnius prolixus. It was also observed that clone Dm28c (Lineage 2) was two to three orders of magnitude more resistant to a lytic agent purified from the crop of $R$. prolixus than the Y strain (Lineage 1). Resistence to lytic activity, as well as reactivity to specific lectins found in the digestive tract of triatomines may provide selective advantages for the development of certain strains of T. cruzi over other strains (cf. Garcia \& Azambuja 1991).

\section{EVOLUTION OF T. CRUZI LINEAGES}

Comparative sequence analysis of the variable region D7 within the LSU rRNA was used to check whether the divergence between $T$. cruzi Lineages 1 and 2 occurred before the divergence of the extant strains as suggested by RAPD (Souto et al. 1996) and also to verify whether the lineages are truly monophyletic. Therefore, sequences of the D7 region were determined for $T$. rangeli and 14 strains of $T$. cruzi and aligned. This alignment was used to build maximum likelihood trees using different transition models and rooted by outgroup (T. rangeli). The tree presented suggests that the divergence between $T$. cruzi Lineages 1 and 2 occurred before the divergence of the extant strains and separate $T$. cruzi into two groups in $100 \%$ of bootstrap replicates (Fig. 2). Also a third group seems to be present which corresponds to group $1 / 2$, where the presence of two types of rDNA cistrons has been shown. Alignment of the sequence of type $1 \mathrm{D} 7$ region of SO3 (group 1/2) places this isolate among Lineage 1 strains (Fig. 2) while type 2 D7 sequence does not allow the establishment of a clear position in the tree. In fact, depending on the algorithm parameters used (parsimony, different models of maximum likelihood and gamma distribution), group $1 / 2$ in some trees seems to be more related to Lineage 1 and in other trees to Lineage 2. Sequence comparisons of small subunit rDNA (SSU) and faster evolving genes will probably help to determine whether group $1 / 2$ is more closely related to Lineage 1 or 2 or if it really is a third lineage of strains.

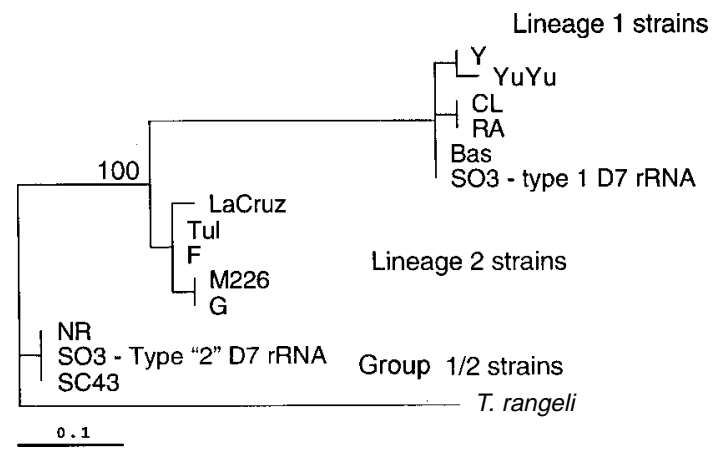

Fig. 2: phylogeny of several Trypanosoma cruzi isolates based on D7 region of the LSU rDNA. T. rangeli was used as an outgroup. The scale bar indicates the number of substitutions per sequence position and the number " 100 " above the node dividing Lineages 1 and 2 indicates that this topology was supported by $100 \%$ bootstrap replicates. This tree inferred by Maximum likelihood has the same topology observed when Parsimony methods were applied but differs from Neighbor Joining trees. In most reconstructions the clade representing group $1 / 2$ could not be resolved. (Bas, Basileu strain; Tul, Tulahuen strain).

Phylogenetic reconstructions using the SSU gene indicate that divergence between the two $T$. cruzi lineages is greater than the distances separating four species of Leishmania and comparable to distances among trypanosomatid genera Crithidia, Leishmania, Endotrypanum and Leptomonas (Briones et al. 1999). Using patristic distances (sum of branch lengths) of the maximum likelihood tree and the evolutionary rates of $0.85 \%$ sequence divergence/100 million years and $2 \%$ sequence divergence/100 million years (Escalante \& Ayala 1995) we estimated the divergence time of the two lineages to be 88 to 37 million years, respectively. 
Because the rate of $2 \%$ sequence divergence $/ 100$ million years for the SSU rDNA might reflect the evolution of faster evoling segments of the molecule we believe that 88 million years of sequence divergence more likely reflects an average rate for the entire gene (Briones et al. 1999).

\section{CONCLUDING REMARKS}

Evidence supporting the existence of two major phylogenetic lineages in T. cruzi is accumulating from independent genomic markers, DNA sequence comparison, population genetics and promoter activity from multigenic families. Data indicate that these lineages are distinct evolutionary units. In addition, epidemiological and biological studies suggest that these lineages have different characteristics. Therefore, the division of $T$. cruzi into two major groups is evident not only genotypically but also physiologically. It is time for the re-evaluation of the T. cruzi taxon and to work out a common nomenclature for these lineages that will serve not only taxonomists but the general community of researchers working with $T$. cruzi. Further genetic characterization of the subgroups within these lineages is a fundamental step towards the understanding of the complex epidemiological and clinical manifestations of Chagas disease.

\section{REFERENCES}

Brenière SF, Bosseno MF, Telleria J, Bastrenta B, Yacsik N, Noireau F, Alcazar JL, Barnabé C, Wicker P, Tibayrenc M 1998. Different behaviour of two Trypanosoma cruzi major clones: transmission and circulation in young bolivian patients. Exp Parasitol 89: 285-295.

Briones MRS, Souto RP, Stolf BS, Zingales B 1999. The evolution of two Trypanosoma cruzi subgroups inferred from rRNA genes can be correlated with the interchange of American mammalian faunas in the Cenozoic and has implications to pathogenicity and host specificity. Mol Biochem Parasitol (in press).

Carreira JCA, Jansen AM, Deane MP, Lenzi HL 1996. Histopathological study of experimental and natural infections by Trypanosoma cruzi in Didelphis marsupialis. Mem Inst Oswaldo Cruz 91: 609-618.

Clark CG, Pung OJ 1994. Host specificity of ribosomal DNA variants in sylvatic Trypanosoma cruzi from North America. Mol Biochem Parasitol 66: 175-179.

Escalante AA, Ayala FJ 1995. Evolutionary origin of Plasmodium and other apicomplexa based on rRNA genes. Proc Natl Acad Sci 92: 5793-5797.

Fernandes O, Sturm NR, Derré R, Campbell DA 1998a. The mini-exon gene: a genetic marker for zymodeme III of Trypanosoma cruzi. Mol Biochem Parasitol 95: 129-133.

Fernandes O, Souto RP, Castro JA, Pereira JB, Fernandes NC, Junqueira ACV, Naiff RD, Barret TV, Degrave W, Zingales B, Campbell DA, Coura JR 1998b. Brazilian isolates of Trypanosoma cruzi from humans and triatomines classified into two lineages using mini-exon and ribosomal RNA sequences. Am J Trop Med Hyg 58: 807-811.

Fernandes O, Santos SS, Junqueira ACV, Jansen AM, Cupolillo E, Campbell DA, Zingales B, Coura JR 1999a. Population heterogeneity of Brazilian Trypanosoma cruzi isolates revealed by mini-exon and ribosomal spacers. Mem Inst Oswaldo Cruz this volume.

Fernandes O, Mangia RH, Lisboa CV, Pinho AP, Morel CM, Zingales B, Campbell DA, Jansen AM 1999b. The complexity of the sylvatic cycle of Trypanosoma cruzi in Rio de Janeiro state (Brazil) revealed by the non-transcribed spacer of the mini-exon gene. Parasitology 118: 161-166.

Floeter-Winter LM, Souto RP, Stolf BS, Zingales B, Buck GA 1997. Can activity of the rRNA gene promoter be used as a marker of speciation? Exp Parasitol 86: 232-234.

Garcia ES, Azambuja P 1991. Development and interaction of Trypanosoma cruzi within the insect vector. Parasitol Today 7: 240-244.

Henriksson J, Aslund L, Macina RA, Franke de Cazzulo BM, Cazzulo JJ, Frasch ACC, Pettersson U 1990. Chromosomal localization of seven cloned antigen genes provides evidence for diploidy and further demonstration of karyotype variability in Trypanosoma cruzi 1990 Mol Biochem Parasitol 42: 213224.

Henriksson J, Pettersson U, Solari A 1993. Trypanosoma cruzi: correlation between karyotype variability and isoenzyme classification. Exp Parasitol 77: 334-348.

Lana M, Silveira Pinto A, Barnabe C, Quesnay V, Noel S, Tibayrenc M 1998. Trypanosoma cruzi: compared vectorial transmissibility of three major clonal genotypes by Triatoma infestans. Exp Parasitol 90: 2025.

Macedo AM, Pena, SDJ 1998. Genetic variability of Trypanosoma cruzi: implications for the pathogenesis of Chagas disease. Parasitol Today 14: 119-124.

Macedo AM, Martins MS, Chiari E, Pena, SDJ 1992. DNA fingerprinting of Trypanosoma cruzi: a new tool for characterization of strains and clones. $\mathrm{Mol}$ Biochem Parasitol 55: 147-154.

Miles MA, Souza A, Povoa M, Shaw JJ, Lainson R, Toye PJ 1978. Isozymic heterogeneity of Trypanosoma cruzi in the first autochtonous patients with Chagas disease in Amazonian Brazil. Nature 272: 819-821.

Miles MA, Lanham SM, De Souza AA, Póvoa M 1980. Further enzymic characters of Trypanosoma cruzi and their evaluation for strain identification. Trans $R$ Soc Trop Med and Hyg 74: 221-242.

Morel C, Chiari E, Camargo E, Mattei D, Romanha A, Simpson L 1980. Strains and clones of Trypanosoma cruzi can be characterized by restriction endonuclease fingerprint of kinetoplast DNA minicircles. Proc Natl Acad Sci USA 88: 1469-1473.

Nunes LR, Carvalho MRC, Shakarian AM, Buck GA 1997a. The transcription promoter of the spliced leader gene from Trypanosoma cruzi. Gene 188: 157168. 
Nunes LR, Carvalho MRC, Buck GA 1997b. Trypanosoma cruzi strains partition into two groups based on the structure and function of the spliced leader and rRNA gene promoters. Mol Biochem Parasitol 86: 211-224.

Oliveira RP, Broude NE, Macedo AM, Cantor CR, Smith CL, Pena SDJ 1998. Probing the genetic population structure of Trypanosoma cruzi with polymorphic microsatellites. Proc Natl Acad Sci USA 95: 37763780.

Pung OJ, Spratt J, Clark CG, Norton TM, Carter J 1998. Trypanosoma cruzi infection of free-ranging liontailed macaques (Macaca silenus) and ring-tailed lemurs (Lemur catta) on St. Catherine's Island, Georgia, USA. Zoo Wildl Med 29: 25-30.

Ruiz RC, Favoretto Jr S, Dorta ML, Oshiro MEM, Ferreira AT, Manque P, Yoshida N 1998. Infectivity of Trypanosoma cruzi strains is associated with differential expression of surface glycoproteins with differential $\mathrm{Ca}^{+2}$ signaling activity. Biochem J 330: 505-511.

Sollner-Webb B, Tower J 1986. Transcription of eukaryotic ribosomal RNA genes. Ann Ver Biochem 55: 801-830.

Souto RP, Zingales B 1993. Sensitive detection and strain classification of Trypanosoma cruzi by amplification of a ribosomal RNA sequence. Mol Biochem Parasitol 62: 45-52.

Souto RP, Fernandes O, Macedo AM, Campbell DA, Zingales B 1996. DNA markers define two major phylogenetic lineages of Trypanosoma cruzi. Mol Biochem Parasitol 83: 141-152.

Stolf BS 1999 Caracterização da Região Promotora do
Cistron de RNA Ribossômico em Duas Linhagens Filogenéticas de Trypanosoma cruzi, MSc Thesis, Universidade de São Paulo, São Paulo.

Stothard JR, Frame IA, Carrasco HJ, Miles MA 1998. On the molecular taxonomy of Trypanosoma cruzi using riboprinting. Parasitology 117: 243-247.

Tibayrenc M 1995. Population genetics of parasitic protozoa and other microorganisms. Adv in Parasitol 36: 48-115.

Tibayrenc M, Ayala F 1988. Isoenzyme variability in Trypanosoma cruzi, the agent of Chagas disease: genetical, taxonomical and epidemiological significance. Evolution 42: 277-292.

Tyler-Cross RE, Short SL, Floeter-Winter LM, Buck GA 1995. Transient expression mediated by the Trypanosoma cruzi rRNA promoter. Mol Biochem Parasitol 72: 23-31.

Yoshida N 1983. Surface antigens of metacyclic trypomastigotes of Trypanosoma cruzi. Infect Immunity 40: 836-839.

Zingales B, Souto RP, Mangia RH, Lisboa CV, Campbell DA, Coura JR, Jansen A, Fernandes O 1998. Molecular epidemiology of American trypanosomiasis in Brazil based on dimorphisms of rRNA and miniexon gene sequences. Int J Parasitol 28: 105-112.

Note added in proof: during this Symposium it was recommended to adopt a common nomenclature to name the two principal groups of Trypanosoma cruzi strains - TC1 and TC2. In this direction, Lineage 1 and Lineage 2 referred in this paper correspond to groups $\mathrm{TC} 2$ and $\mathrm{TC} 1$, respectively. 